\title{
Antiferroelectric Liquid Crystals with Induced Intermediate Polar Phases and the Effects of Doping with Carbon Nanotubes
}

\author{
Jan P. F. Lagerwall ${ }^{1}$, Roman Dabrowski ${ }^{2}$, Giusy Scalia ${ }^{3}$ \\ ${ }^{1}$ University of Stuttgart, Institute of Physical Chemistry, 70569 Stuttgart, Germany \\ ${ }^{2}$ Military University of Technology, 00-908 Warsaw, Poland \\ ${ }^{3}$ Max Planck Institute for Solid State Research, Synthetic Nanostructures Group, 70569 Stuttgart, Germany
}

\begin{abstract}
By mixing a commercial broad-temperature-range nematic liquid crystal mixture with a single-component antiferroelectric chiral smectic exhibiting two different chiral smectic-C-type phases as only mesophases, we have induced three phases which appear in neither of the two components; the paraelectric SmA* phase and the so-called intermediate phases $\mathrm{SmC}_{\beta}{ }^{*}$ and $\mathrm{SmC}_{*}^{*}$, antiferroelectric and helielectric in nature, respectively. The generation of the two latter phases in mixtures where one component is an essentially non-chiral nematic is highly unexpected, since these phases are generally linked to high degree of smectic order and / or strong chiral interactions. It is probably made possible through microphase segregation driven by the incompatibility of the fluorinated tail of the smectic component with the non-fluorinated constituents of the nematic mixture. We also doped the nematic with single-wall carbon nanotubes (SWCNTs) before adding it to the smectic at the same concentration, allowing us to study the effect of SWCNTs on antiferroelectric liquid crystals. Although the final SWCNT concentration was very small $(0.002 \mathrm{wt.}$ - $\%)$ the phase sequence was radically altered, the ordinary $\mathrm{SmC}^{*}$ phase now being present all the way between $\mathrm{SmA}^{*}$ and crystallization, while all other variations of smectic-C-type order were suppressed.
\end{abstract}

PACS codes: 77.84.Nh, 61.46.+w, 64.70.Md

Keywords: Liquid crystal, Dielectric spectroscopy, Smectic, Chiral, Antiferroelectric, Carbon nanotube, Doping

\section{Introduction}

Smectic liquid crystals exhibit a layered structure with liquid-like short-range positional correlation within the layers, but quasi-long-range translational order along the layer normal [1]. They can thus be regarded as $1 \mathrm{D}$ crystals or $2 \mathrm{D}$ liquids at the same time. When the director (the average direction of the

\footnotetext{
${ }^{1}$ E-mail for correspondence: jan.lagerwall@1csoftmatter.com
} 
main molecular symmetry axis) is tilted with respect to the layer normal (smectic-C-type order) and the phase is chiral, these liquid crystals (LCs) exhibit a meso- or macroscopic spontaneous electric polarization $\mathbf{P}_{\mathbf{s}}$ [2], rendering them heli-, ferro- or antiferroelectric depending on the exact phase type and the sample geometry [3-4]. Such LCs can exhibit very complex phase sequences with at least five variations of the basic tilted $\mathrm{SmC}^{*}$ phase having been identified [1, 5-9]. While the richest phase sequence, including the so-called intermediate chiral smectic-Ctype phases $\mathrm{SmC}_{\beta}^{*}$ and $\mathrm{SmC}_{*}^{*}$ (antiferroelectric and helielectric in nature, respectively $[1,4]$ ), initially was observed only in single-component antiferroelectric liquid crystals (AFLCs) of very high purity [8-9], recent work has demonstrated that the complete chiral smectic-C-type phase sequence can be induced, often over a much broader temperature range, by strategic mixing [10-12].

Here we give a new example of this phenomenon, unique in the sense that one component in the mixture is strictly nematic (i.e. exhibiting orientational but no translational order) and essentially non-chiral. This is remarkable since our current understanding of the intermediate phases is that they require high degree of smectic translational order $[8,11]$ and / or strong chiral interactions [5,9]. The most fundamental requirement for their formation, however, seems to be a frustration between incompatible types of LC organization [1, 12 ], a condition which is easily met in the present case. Moroever, we propose that also the requirement on high smectic order is fulfilled thanks to microphase segregation driven by the incompatibility between a partially fluorinated achiral end chain of the smectic molecule with the non-fluorinated constituents of the nematic mixture.

During the last five years there has been a considerable interest in the combination of carbon nanotubes (CNTs) and liquid crystals. On the one hand, lyotropic as well as thermotropic LCs can be used to align and at the same time disperse CNTs [13-19], on the other, the CNTs influence the properties of the LC host and it has been suggested that CNT-doping in this way can enhance the properties of LC mixtures for use in displays [20-24]. All studies so far were made using nematic LCs. Here we for the first time extend the investigations to CNT-doping of chiral smectics, addressing several interesting new questions relevant to this combination of hard nanoparticle guest and soft matter host. Most importantly, it is not obvious how the CNTs are incorporated in the smectic structure (are they aligned along the layer normal, along the director or are they not aligned at all by the smectic host ?) and what impact the nanotubes have on the smectic order and / or the director field, the smallscale variations of which can be considerably more complex in smectics than in nematics [1]. Our studies, using dielectric spectroscopy with simultaneous texture monitoring, demonstrate that the presence of CNTs, even at very small concentration, has immense impact on the phase sequence of the mixture.

\section{Materials and methods}

The basic AFLC compound, in this paper referred to as AF0, is shown together with its phase sequence in Figure 1. This was mixed with the commercial multi-component mixture RO-TN-403/015 S from Hoffmann-La Roche, developed for use in twisted nematic (TN) displays and exhibiting a nematic phase from below room temperature to about $80^{\circ} \mathrm{C}$. Its exact composition is not known to us but the constituents are [25] alkyl cyano biphenyls, alkoxy cyano biphenyls, alkyl cyano terphenyls, cyanophenyl pyrimidines, terpyrimidines and $0.1 \mathrm{wt}$.$\%$ cholesteryl nonaoate. The latter is a weak chiral dopant added in order to ensure a uniform twisted sense when the mixture is used in a $\mathrm{TN}$ device. The amount is so small, however, that the mixture for all practical purposes can be treated as non-chiral.

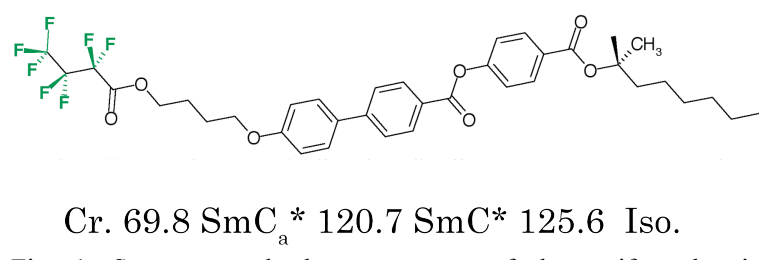

Fig. 1. Structure and phase sequence of the antiferroelectric compound (in the following referred to as AF0) used in the mixtures.

The CNTs were HiPCO single-wall carbon nanotubes (SWCNTs) purchased from CNI, with a 
nominal purity of $>95 \%$. The diameter of $\mathrm{HiPCO}$ SWCNTs is about $1 \mathrm{~nm}$ and the length typically on the order of 100-200 nm [26].

The dispersion of CNTs is a non-trivial task and much research efforts are currently devoted to optimizing the procedures and better understand the mechanisms involved. As an efficient dispersion of CNTs requires relatively large amounts of a liquid host it was impossible to introduce the CNTs directly into AF0, which has a melting point of $\sim 70^{\circ} \mathrm{C}$ and the total available amount of which was some $50 \mathrm{mg}$. Instead we used the nematic mixture as an initial host for CNT dispersion, as this was available in large quantities and as nematic thermotropic LCs with aromatic molecule cores have been shown to be good hosts for dispersing CNTs [19].

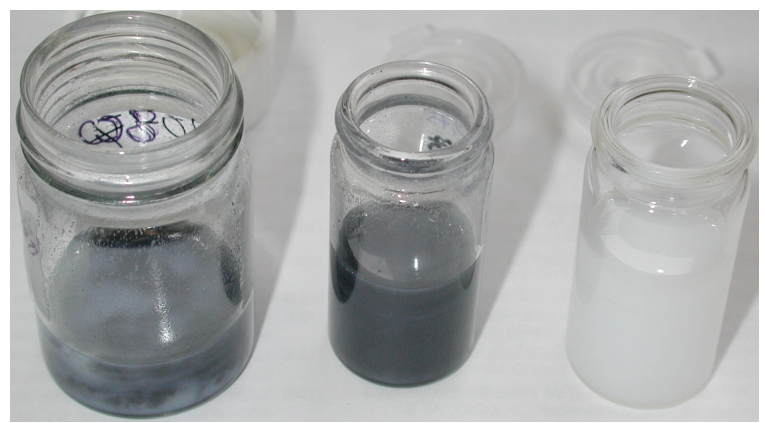

Fig. 2. The nematic mixture RO-TN-403/015 S, pure (right) and doped with CNTs at a concentration of $0.02 \mathrm{wt} .-\%$, five weeks after mixture preparation. The sample on the far left was sonicated with tip sonicator while the middle sample was gently stirred over a weekend, giving much better long-term dispersion stability.

Normally, the dispersion of CNTs requires highpower ultrasonication with a tip sonicator dipped into the mixture, but this turned out not to be the optimal method in our case. Figure 2 shows, in addition to the pure nematic liquid crystal on the right, two samples containing CNTs at 0.02 wt.- $\%$ concentration five weeks after preparation. In the left bottle we had dispersed the CNTs using a tip sonicator, while we for the middle bottle used only gentle stirring with a magnetic stir bar over a weekend. It is clear that the long-term stability is much better in the latter case, still being completely uniform. The tip-sonicated sample, in contrast, had obviously phase separated into CNT-rich dark and CNT-poor light grey volumes. Neither preparation rendered bundle-free mixtures but the bundle size was relatively small and the uniformity good in the gently stirred mixture. The complete characterization of the CNT-doped nematic LC will be described elsewhere.

We initially dispersed $0.73 \mathrm{mg}$ CNTs in $4.1 \mathrm{~g}$ of the nematic mixture. After stirring until uniform, this mixture was further diluted to a concentration of 0.01 wt.-\% of SWCNTs, before it was added to AF0, giving the final CNT-containing AFLC mixture a nanotube concentration of 0.002 wt.-\%. Since our way of introducing CNTs into smectics was via the nematic host, a fundamental requirement on the smectic material was that it can accept considerable amounts of the nematic mixture without losing its interesting tilted smectic-C-type phases. While many smectic compounds tested lost all tilted phases already at very small concentration of the nematic mixture, we could add more than $20 \%$ of RO-TN403/015 S to AF0, exhibiting a direct transition between the isotropic liquid and the tilted $\mathrm{SmC}^{*}$ phase, and still get tilted smectic phases. This molecule was thus an excellent AFLC choice for our studies.

For the dielectric spectroscopy experiments, carried out using an HP4192A impedance bridge, the mixtures were filled into standard LC test cells (MC2, Chalmers, Gothenburg, Sweden). The glass substrates of the cells were equipped with transparent ITO electrodes $(100 \mathrm{~nm}$ thickness), for the application of electric fields, and rubbed polyimide (20 nm thickness), ensuring the uniform planar alignment of the liquid crystal. The cell thickness was $23.5 \mu \mathrm{m}$. During the measurement the cells were kept in a temperature-regulated compartment (a Julabo FP-4 circulator was used for temperature control) mounted on an Olympus $\mathrm{BH}-2$ polarizing microscope. This was equipped with a standard USB webcam, allowing us to monitor the texture of the sample throughout the measurement via our measurement software (DiScO, FLC Electronics, Gothenburg, Sweden).

\section{Results and discussion}

\subsection{The CNT-free AFLC mixture}

Fig. 3 shows the dielectric absorption spectrum, obtained on heating, of AF0. It is easy to recognize 
the high-temperature helielectric $\mathrm{SmC}^{*}$ phase [4] (plotted in yellow) through its characteristic strong dielectric absorption at relatively low frequencies. This is related to the field-induced distortion of the natural helical structure of the phase, taking place because of the coupling between the measuring field and the spontaneous polarization $\mathbf{P}_{\mathrm{s}}$, directed perpendicular to the smectic-C tilt plane which precesses in a spiral-like fashion along the smectic layer normal [1-2]. The low-temperature phase is the anticlinic and antiferroelectric $\mathrm{SmC}_{\mathrm{a}}{ }^{*}$ phase (plotted in purple), exhibiting essentially no dielectric response since $\mathbf{P}_{\mathrm{s}}$ is cancelled out locally on the scale of every two smectic layers [1].

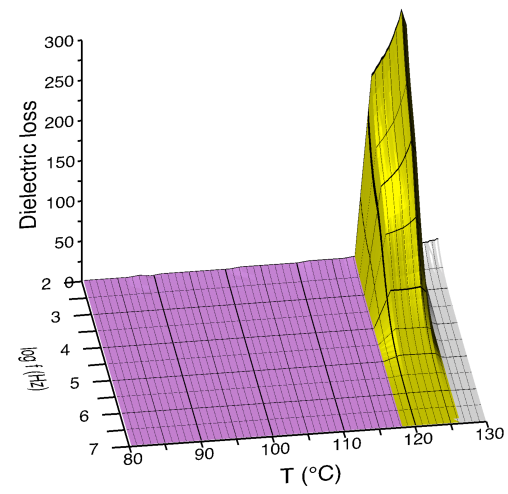

Fig. 3. Dielectric absorption spectrum of pure AF0, exhibiting the helielectric $\mathrm{SmC}^{*}$ phase (yellow) and the antiferroelectric $\mathrm{SmC}_{\mathrm{a}}{ }^{*}$ phase (purple) below the isotropic liquid (grey). Measuring field $10 \mathrm{mV} / \mu \mathrm{m}$, cell gap $23.5 \mu \mathrm{m}$.

Surprisingly, the mixtures with the nematic component turned out not only to display the original AF0 phases, but three further smectic phases could be generated. The richest phase sequence was found at about 17.5 wt.-\% RO-TN-403/015 S, cf. Fig. 4, and all further experiments were thus carried out on mixtures with this composition, which we in the following will refer to as AF1. The dielectric spectroscopy measurements on AF1 had to be carried out with very weak measuring field $(2 \mathrm{mV} / \mu \mathrm{m})$, as strong non-linear effects otherwise distorted the spectra. This was in strong contrast to AF0, with which a measurement field of $10 \mathrm{mV} / \mu \mathrm{m}$ produced no artifacts.

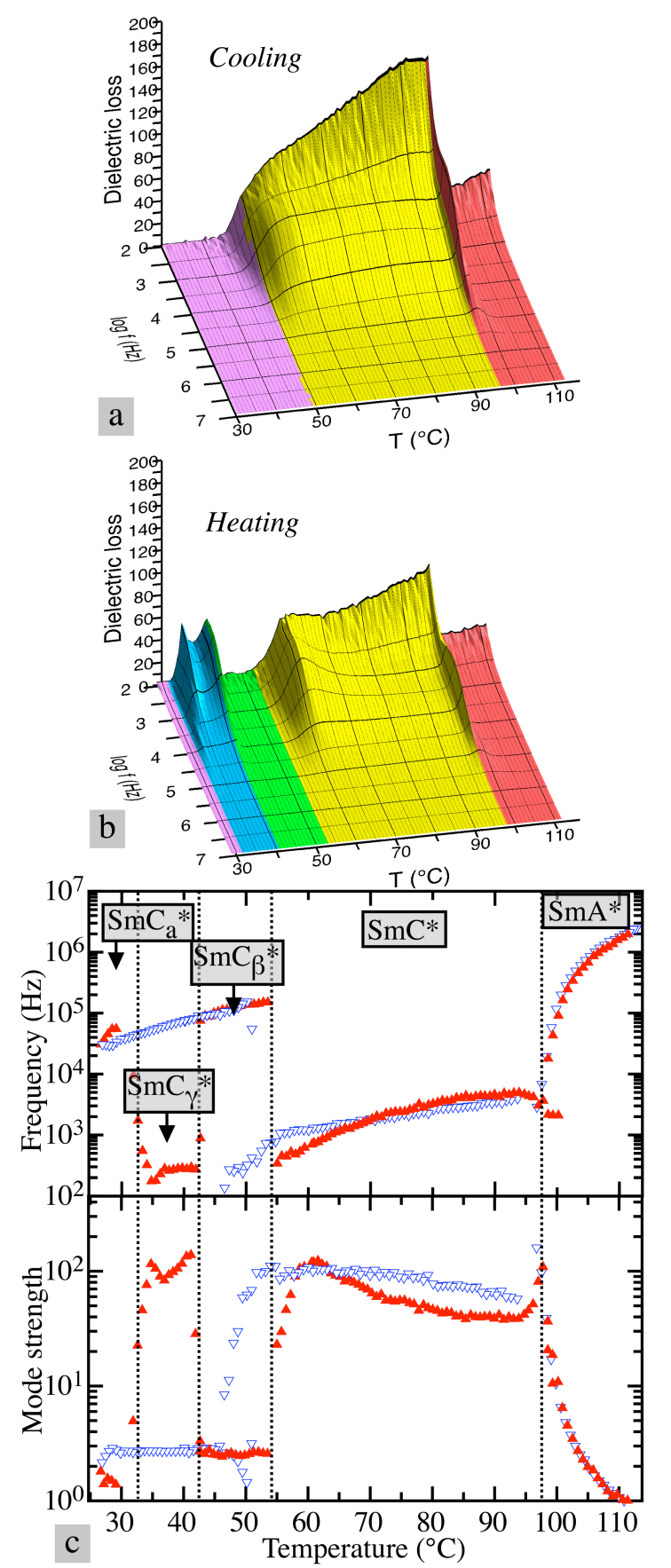

Fig. 4. Dielectric absorption spectra on cooling (a) and on subsequent heating (b) of the mixture AF1, and the results from fitting a Cole-Cole function to the data (c) where red upward triangles refer to the heating and blue downward triangles to the cooling runs, respectively. The phases indicated in (c) refer to heating. Measuring field $2 \mathrm{mV} / \mu \mathrm{m}$, cell gap $23.5 \mu \mathrm{m}$. 
Between the clearing point and $\sim 97^{\circ} \mathrm{C}$ the AF1 mixture exhibited the paraelectric non-tilted SmA* phase, present in neither of the components on their own. In the dielectric spectrum this is easily recognized by its characteristic soft mode, exhibiting critical slowing-down and diverging strength on cooling towards the tilting transition. At about $97^{\circ} \mathrm{C}$, on cooling as well as on heating, the transition to the tilted and helielectric SmC* phase took place. The dielectric response was weaker than in the case of pure AF0, a result of the mixture containing one fifth non-chiral molecules with essentially no lateral dipole moment. Moreover, the $\mathrm{SmC}^{*}$ phase temperature is considerably broadened from the $\sim 8^{\circ} \mathrm{C}$ in pure AF0 to more than $40^{\circ} \mathrm{C}$ in AF1. At about $50^{\circ} \mathrm{C}$ the $\mathrm{SmC}^{*}$ phase is replaced by an antiferroelectric phase giving no dielectric response in the cooling measurement, and no further phase transition is observed down to room temperature. When the sample was reheated, however, a transition to a polar phase took place at just above $30^{\circ} \mathrm{C}$, which lasted for about $10^{\circ} \mathrm{C}$, where another antiferroelectric phase took its place. This was then replaced by $\mathrm{SmC}^{*}$ at $\sim 55^{\circ} \mathrm{C}$. The two new phases observed only on heating are clearly the intermediate phases $\mathrm{SmC}_{*}^{*}$ and $\mathrm{SmC}_{\beta} *[1,5-9]$.

The ordinary antiferroelectric $\mathrm{SmC}_{\mathrm{a}} *$ phase cannot be distinguished from the intermediate antiferroelectric $\mathrm{SmC}_{\beta}{ }^{*}$ phase in the dielectric spectrum, as neither gives any polar response. The single weak dielectric mode observed in both phases, exhibiting an Arrhenius-type temperature dependence of the limiting frequency (cf. Fig. 4c), is a noncollective mode, corresponding to rotation of the molecules around their short axes. The question thus arises which antiferroelectric phase was actually formed below $\mathrm{SmC}^{*}$ on cooling. On the one hand, the phase transformation from $\mathrm{SmC}^{*}$ was over at about $45^{\circ} \mathrm{C}$, which is within the temperature range of $\mathrm{SmC}_{\beta} *$ on heating. On the other, no transition was observed on further cooling, but on reheating the transition to $\mathrm{SmC}^{*}$ took place, suggesting that the system had gone directly from $\mathrm{SmC}^{*}$ to $\mathrm{SmC}_{\mathrm{a}} *$ in the cooling experiment. This was supported by an investigation of the sample textures recorded during the cooling experiment. The striped texture which is characteristic of the intermediate phases [8], reflecting their long-pitch helical structure, was observed only briefly during the transition from $\mathrm{SmC}^{*}$, while the texture of the antiferroelectric phase had no trace of stripes and thus resembled that of the $\mathrm{SmC}_{\mathrm{a}} *$ phase, generally with much shorter pitch. The exact reason for the difference in phase sequence on cooling and heating is not clear at the moment, but it is well known that the enthalpies of the transitions between different chiral smectic-C-type phases are very small, that very strong supercooling and superheating effects can occur and that the phases can disappear from the phase diagram as a result of quite subtle disturbances [8-9,11].

While some cases where the intermediate phases have been induced by mixing are known [10-12] it is a more common phenomenon that they disappear as a result of mixing. Their appearance has been linked to a high degree of smectic order [8,11] and / or strong chiral interactions $[5,9]$. As one of the components in the present case is practically non-chiral and exhibits only a nematic phase, i.e. the extreme in low smectic order, the induction of the intermediate phases is very surprising. A possible explanation may be found in the fluorinated tail of the antiferroelectric chiral smectic compound AFo (Fig. 1). None of the constituents of the nematic mixture has a fluorinated tail, hence the incompatibilities between the fluorinated and non-fluorinated components may lead to microphase segregation [1], increasing the smectic order and thereby allowing the generation of the intermediate phases. Equally important is the strong tendency to form tilted smectic phases of AF0, in which the SmC* phase forms directly out of the isotropic liquid. This is evidenced by the fact that several other chiral smectics, exhibiting a SmA* phase at high temperatures, lost the tilted phases completely when mixed with the nematic, already at low concentration. Also in our previous study of induced smectic phases involving an achiral nematicforming component [11] the chiral smectic component had very strong tendency for tilt and exhibited no $\mathrm{SmA}^{*}$ phase.

\subsection{The CNT-doped AFLC mixture}

The CNT-containing AFLC mixture corresponding to AF1, in the following referred to as AF1cnt, was produced following the same procedure as for AF1, but now the nematic phase contained $0.01 \mathrm{wt} .-\%$ of HiPCO SWCNTs. When added to AF0 at 17.5 wt.-\% 

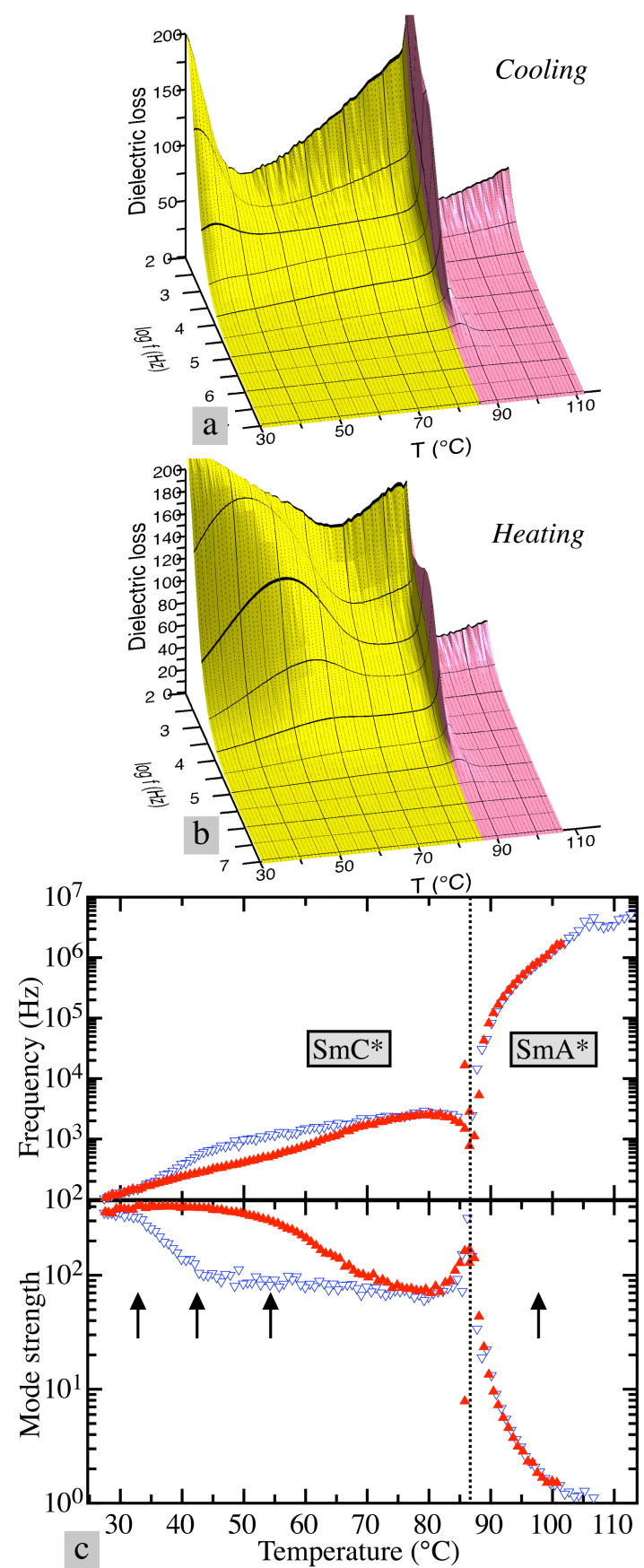

Fig. 5. Dielectric absorption spectra on cooling (a) and on subsequent heating (b) of the mixture AF1cnt, and the results from fitting a Cole-Cole function to the data (c) where red upward triangles refer to the heating and blue downward triangles to the cooling runs, respectively. The arrows in (c) indicate the phase transitions observed on heating the corresponding CNT-free mixture AF1. Measuring field $2 \mathrm{mV} / \mu \mathrm{m}$, cell gap $23.5 \mu \mathrm{m}$. concentration, this yielded a final CNT concentration of 0.002 wt.- $\%$ in AF1cnt. In cells filled with the CNT-containing smectic mixture, the nanotubes had a stronger tendency to aggregate than in the nematic host, seen through the presence of relatively large bundles (several microns in diameter). This does not necessarily mean that the smectic host is worse in incorporating the CNTs, but it could also be related to the fact that the mixture had to be heated into the isotropic phase for filling it into the cells, whereas the CNT-containing nematic mixture could be filled in the nematic phase. There was at least no aggregation of bundles in any special area of the AF1cnt sample, but the distribution of CNTs was acceptably uniform throughout the sample.

Despite the very small amount of CNTs, the effect on the phase sequence on the mixture is strong, as seen in the dielectric spectra, plotted in Fig. 5 with the same scaling as in Fig. 4. We first note that the tilting transition is pushed further downwards by about $10 \mathrm{~K}$. Second, the soft mode at the tilting transition has become considerably stronger, reaching a peak value of more than 300, about twice the maximum value measured without CNTs (the scaling in Figs. 4 and 5 is identical to facilitate comparison). While the $\mathrm{SmC}^{*}$ response close to the tilting transition is similar to that of the measurement without CNTs on cooling, it is now just as strong on heating, yielding the heating response about 50\% stronger compared to the data obtained without CNTs. The most conspicuous difference is however the absence of any other smectic-C-type phases than $\mathrm{SmC}^{*}$, on cooling as well as on heating, and the consequent broadening of the $\mathrm{SmC}^{*}$ phase. When in the initial cooling measurement AF1cnt reached the temperature at which AF1 exhibited the $\mathrm{SmC}^{*}$ $\mathrm{SmC}_{\beta} *$ transition on heating, the dielectric response grew considerably in strength and the frequency dropped somewhat. In the subsequent heating run, the strong $\mathrm{SmC}^{*}$ response stayed unaltered up to about $50^{\circ} \mathrm{C}$, about the temperature where $\mathrm{AF} 1$ went from $\mathrm{SmC}_{\beta}^{*}$ to $\mathrm{SmC}^{*}$ in the corresponding heating measurement.

The strength of the helielectric mode is proportional to the squares of the spontaneous polarization and of the pitch [27], hence the increase could be related to an increase in either of these parameters. When looking at the textures photographed during the measurement, we noticed 
that distinct broadly spaced stripes reflecting a longpitch helical structure developed at about $40^{\circ} \mathrm{C}$ on cooling. These stripes remained during heating up to about $70^{\circ} \mathrm{C}$, hence the additional strong response can be attributed to fluctuations in a very long-pitch structure. Considering that the striped texture is a feature of the intermediate $\mathrm{SmC}_{*}{ }^{*}$ and $\mathrm{SmC}_{\beta} *$ phases of $\mathbf{A F 1}$, and that there is a certain correspondence between its temperature range in AF1cnt and that of the intermediate phases in AF1, one may suggest that the intermediate phases are actually not completely absent in the CNT-containing mixture, but that they in some sense coexist with the $\mathrm{SmC}^{*}$ phase, but further studies are required in order to elucidate this.

It is too early to give a complete explanation for the strong sensitivity to the presence of CNTs but we can give some educated guesses as to the origin. We can first of all expect that the CNTs, very long compared to the thickness of a smectic layer, will have strong impact on the director variation from layer to layer. The CNTs can promote only synclinic order (same tilting direction of the director in adjacent layers) since the nanotube direction will not change at all on the length scale of a smectic layer. Thus, they promote the ordinary (synclinic) $\mathrm{SmC}^{*}$ phase but tend to suppress any other variation of chiral smectic-C-type order seen in AF1, all comprising anticlinic layer boundaries [1]. Furthermore, when the CNTs are in bundles they must be expected to have a generally disordering effect on the host phase, particularly devastating for any type of regular microphase segregation. Thus, in bundles as well as in a well-dispersed state we should expect the CNTs to promote ordinary $\mathrm{SmC}^{*}$-or even $\mathrm{SmA}^{*}$ - at the cost of its variations. If the great majority of the CNTs are confined to relatively large bundles we can finally expect two types of LC structural domains in the sample; in the vicinity of CNT bundles the order is strongly disturbed and only $\mathrm{SmC}^{*}$ prevails, but between bundles there would be little if any nanotubes present, allowing for the natural phase sequence of the AF1 mixture to develop here. This could be an explanation to the signs of phase coexistence that were observed in our experiments.

Fig. 6 shows the magnitude of the spontaneous polarization of AF1 and AF1cnt as a function of temperature. Unfortunately, the high switching threshold as AF1 entered the antiferroelectric phase prevented measurements below this temperature, hence the data is somewhat inconclusive. However, it seems that the saturation values are relatively similar with and without CNTs whereas the temperature dependence just below the SmA* phase differs slightly between the two cases. In any case, the differences are too small to explain the differences in the dielectric response at low temperatures, which must rather be related to changes in the pitch of the helical modulation.

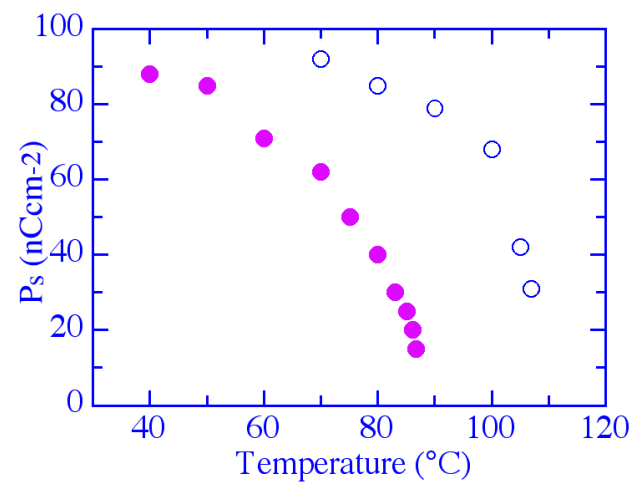

Fig. 6. The magnitude of spontaneous polarization of AF1 (empty symbols) and AF1ent (filled symbols) as a function of temperature.

\section{Conclusions and outlook}

By using a nematic LC host as an initial dispersing medium, subsequently mixed with an antiferroelectric chiral smectic compound, we have successfully doped an antiferroelectric liquid crystal with single-wall carbon nanotubes (SWCNTs). Whilst the mixture of the nematic component with the chiral smectic compound induced an unexpectedly rich polymesomorphism in the absence of nanotubes, in particular exhibiting induced intermediate $\mathrm{SmC}_{\beta} *$ and $\mathrm{SmC}_{\gamma}^{*}$ phases, the CNTcontaining mixture exhibited only the helielectric SmC* phase in addition to the paraelectric $\mathrm{SmA}^{*}$ phase. However, its behavior at low temperatures, as studied by dielectric spectroscopy combined with texture monitoring, exhibits some anomalies, possibly suggesting a complex coexistence of phases. 
We propose that the rich phase sequence in the CNTfree case reflects mainly a frustration between opposing types of LC organization and is made possible through microphase segregation between the fluorinated chiral smectic mesogens and the nonfluorinated constituents of the nematic mixture. When adding the greatly extended CNTs these should strongly promote synclinic order and they might disrupt the smectic organization in general, in particular if in bundles, explaining the radical change in phase sequence observed on CNT-doping

With this study we have started addressing a number of interesting questions regarding nanoparticle doping of liquid crystals with higher degree of order. We have demonstrated that CNTs can have a very strong impact on the phase sequence of AFLCs, even at very low concentration, without necessarily modifying the properties of each phase very much. Many interesting questions remain to be addressed, for instance how the CNTs are incorporated in the smectic phase. We hope to be able to answer these questions after having improved the CNT dispersion quality in the smectic phase and carried out a series of investigations using different experimental techniques specifically selected for the task, e.g. polarized Raman spectroscopy.

\section{Acknowledgments}

We are grateful to F. Giesselmann and S. Roth for their continuous support and encouragement. Financial support from the Knut and Alice Wallenberg foundation (JL) and the Marie Curie Intra-European Fellowship Program (GS) is gratefully acknowledged.

\section{References}

[1] J.P.F. Lagerwall, F. Giesselmann, Chemphyschem 7 (2006) 20

[2] R.B. Meyer, L. Liebert, L. Strzelecki, P. Keller, J. Phys. (Paris) Lett. 36 (1975) L69

[3] N.A. Clark, S.T. Lagerwall, Appl. Phys. Lett. 36 (1980) 899
[4] J.P.F. Lagerwall, Phys. Rev. E 71 (2005) 051703

[5] M. Cepic, E. Gorecka, D. Pociecha, B. Zeks, H.T. Nguyen, J. Chem. Phys. 117 (2002) 1817

[6] L.S. Hirst, S.J. Watson, H.F. Gleeson, P. Cluzeau, P. Barois, R. Pindak, J. Pitney, A. Cady, P.M. Johnson, C.C. Huang, A.M. Levelut, G. Srajer, J. Pollmann, W. Caliebe, A. Seed, M.R. Herbert, J.W. Goodby, M. Hird, Phys. Rev. E 65 (2002) 041705

[7] H.F. Gleeson, L.S. Hirst, Chemphyschem 7 (2006) 321.

[8] J.P.F. Lagerwall, P. Rudquist, S.T. Lagerwall, F. Giesselmann, Liq. Cryst. 30 (2003) 399

[9] E. Gorecka, D. Pociecha, M. Cepic, B. Zeks, R. Dabrowski, Phys. Rev. E 65 (2002) 061703

[10] S. Jaradat, N.W. Roberts, Y. Wang, L.S. Hirst, H.F. Gleeson, J. Mater. Chem. 16 (2006) 3753

111] J.P.F. Lagerwall, F. Giesselmann, C. Selbmann, S. Rauch, G. Heppke, J. Chem. Phys. 122 (2005) 144906.

[12] J.P.F. Lagerwall, G. Heppke, F. Giesselmann, Eur. Phys. J. E 18 (2005) 113

[13] M.D. Lynch, D.L. Patrick, Nano. Lett. 2 (2002) 1197.

[14] I. Dierking, G. Scalia, P. Morales, D. LeClere, Adv. Mater. 16 (2004) 865.

[15] I. Dierking, G. Scalia, P. Morales, J. Appl. Phys. 97 (2005) 044309

[16] G. Scalia, M. Haluska, U. DettlaffWeglikowska, F. Giesselmann, S. Roth, AIP Conf. Proc. 786 (2005) 114

[17] J.P.F. Lagerwall, G. Scalia, M. Haluska, U. Dettlaff-Weglikowska, S. Roth, F. Giesselmann, Adv. Mater. (2006) in press

[18] J.P.F. Lagerwall, G. Scalia, M. Haluska, U. Dettlaff-Weglikowska, S. Roth, F. Giesselmann, Physica Status Solidi B (2006) in press (on-line DOI: 10.1002/pssb.200669146)

[19] G. Scalia, J.P.F. Lagerwall, M. Haluska, U. Dettlaff-Weglikowska, F. Giesselmann, S. Roth, Physica Status Solidi B (2006) in press (on-line DOI: 10.1002/pssb.200669205)

[20] I.S. Baik, S.Y. Jeon, S.H. Lee, K.A. Park, S.H. Jeong, K.H. An, Y.H. Lee, Appl. Phys. Lett. 87 (2005) 263110.

[21] W. Lee, Y.C. Shih, Journal of the Society for Information Display 13 (2005) 743.

[22] W. Lee, J.S. Gau, H.Y. Chen, Applied Physics B-lasers and Optics 81 (2005) 171. 
[23] W. Lee, C.Y. Wang, Y.C. Shih, Appl. Phys. Lett. 85 (2004) 513.

[24] H.Y. Chen, W. Lee, Appl. Phys. Lett. 88 (2006) 222105.

[25] P. Gerber, Applied Physics A - Materials Science \& Processing 26 (1981) 139.

[26] M.F. Islam, E. Rojas, D.M. Bergey, A.T. Johnson, A.G. Yodh, Nano. Lett. 3 (2003) 269

[27] A. Levstik, T. Carlsson, C. Filipic, I. Levstik, B. Zeks, Phys. Rev. A 35 (1987) 3527 